\title{
Identificação de dificuldades no início do aleitamento materno mediante aplicação de protocolo
}

\author{
Identification of difficulties at the beginning of breastfeeding by means of protocol application
}

\author{
Maria Antonieta de Barros Leite Carvalhaes ${ }^{2}$, Cláudia Regina Hostin Corrêa ${ }^{2}$
}

\section{Resumo}

Objetivo: dimensionar o grupo de mães/recém-nascidos com necessidades especiais de apoio para um início bem sucedido do aleitamento materno, mediante aplicação de protocolo preconizado pelo UNICEF, e verificar práticas assistenciais associadas com dificuldades no aleitamento materno.

Metodologia: trata-se de um estudo transversal, descritivo. A amostra foi constituída de 50 binômios mãe/recém-nascido, selecionados mediante sorteio, em maternidade que atende ao parto de baixo risco pelo SUS. Utilizando protocolo para observação e avaliação de mamada, foram registrados os comportamentos de cada dupla, computando-se a frequiência de comportamentos desfavoráveis ao aleitamento materno. A seguir, foram criados escores (bom, regular, ruim) para avaliar cada aspecto da mamada observada. Investigou-se também a associação entre determinadas práticas assistenciais e escores desfavoráveis. Adotou-se p $<0,05$ como nível crítico.

Resultados: a frequiência de binômios que apresentaram comportamentos sugestivos de sérias dificuldades (escore ruim) com o início do aleitamento materno variou entre $2 \%$ e $22 \%$, conforme o aspecto da mamada avaliada. As dificuldades mais presentes foram a má posição corporal da mãe e do bebê durante a mamada e a inadequação da interação mãe/neonato. Tais dificuldades foram significativamente mais freqüentes quando o parto foi cirúrgico $(\mathrm{p}<$ 0,05 ). O uso de fórmula láctea e/ou soro glicosado também associouse com piores escores em alguns aspectos da mamada.

Conclusão: a aplicação do protocolo para a observação e avaliação de mamada identificou alta prevalência de binômios mãe/ bebê com comportamentos sugestivos de dificuldades com o início da amamentação, em especial quando o parto foi cirúrgico e quando foram oferecidos suplementos ao neonato.

J Pediatr (Rio J) 2003;79(1):13-20: aleitamento materno, comportamento materno, comportamento do lactente, assistência perinatal.

1. Professora Assistente Doutora, Departamento de Enfermagem, Faculdade de Medicina de Botucatu - UNESP.

2. Graduada em Enfermagem, Faculdade de Medicina de Botucatu UNESP.

Artigo submetido em 24.04.02, aceito em 23.10.02.

\begin{abstract}
Objective: to assess a group of mothers/newborns with the necessity of special support at the beginning of breastfeeding by means of protocol application recommended by UNICEF and to verify assisting practices associated with difficulties of breastfeeding.

Methods: in this descriptive study, the sample comprised 50 mother/newborn pairs randomly selected in a maternity where low risk deliveries are care by SUS (Brazilian Unified Health System). The breastfeeding observation protocol was used to record the behavior of each pair, including the frequency of negative behavior regarding breastfeeding. Next, each aspect was scored as good, regular or poor. The association between negative scores and particular assisting practices was also investigated. A critical level of $p<0.05$ was used.

Results: the frequency of pairs presenting evidence of severe problems (poor score) at the beginning of breastfeeding ranged from $2 \%$ to $22 \%$ according to the aspects assessed. The most frequently observed difficulties were mother and infant's bad positioning during breastfeeding and inappropriate mother/newborn interaction. These problems were significantly more frequent after surgical deliveries ( $\mathrm{p}<0.05$ ). Milk formula and/or glucose solution was also associated with the worst scores in some breastfeeding aspects.

Conclusion: the protocol application to observe and evaluate breastfeeding identified a high prevalence of mothers/newborn pairs with difficulties to begin breastfeeding, especially when the delivery was surgically performed and when the newborn was offered supplementary liquids.
\end{abstract}

J Pediatr (Rio J) 2003;79(1):13-20: maternal breastfeeding, maternal behavior, infant behavior, perinatal care.

Apesar dos avanços nas taxas de aleitamento materno observados na última década, a situação do aleitamento materno no Brasil ainda está longe da preconizada pela Organização Mundial da Saúde: amamentação exclusiva até o sexto mês e aleitamento materno com alimentos 
complementares até dois anos ou mais de idade ${ }^{1}$. Estudo populacional realizado em 1995, no município onde este estudo foi desenvolvido, detectou um padrão insatisfatório de aleitamento materno; aos trinta dias, somente $29 \%$ das crianças eram exclusivamente amamentadas, e aos quatro meses, apenas $4,6 \%{ }^{2}$.

A duração do aleitamento materno pode ser favorecida ou restringida por fatores biológicos, culturais, relativos à assistência à saúde e socioeconômicos ${ }^{3}$. Os profissionais de saúde, por meio de suas atitudes e práticas, podem influenciar positiva ou negativamente o início da amamentação e sua duração. Em particular, as enfermeiras e os obstetras, durante o período pré-natal, e os pediatras e a equipe de enfermagem, no período neonatal, podem incentivar a amamentação e apoiar as mães, ajudando-as a iniciála precocemente e a adquirir autoconfiança em sua capacidade de amamentar ${ }^{4-8}$. O desenvolvimento ostensivo de ações de apoio à amamentação dentro das instituições hospitalares que assistem ao parto e ao recém- nascido é reconhecido como medida capaz de modificar o perfil do aleitamento materno em uma população ${ }^{1,3,9,10}$.

Dentre as ações de apoio ao aleitamento materno recomendadas, encontra-se a observação de cada dupla mãe/ neonato durante uma mamada. Essa atividade tem sido proposta como forma de identificar mães e bebês que necessitam de apoio extra ${ }^{9,11}$, tendo sido proposto pelo UNICEF um protocolo para orientar essa atividade. Nele são apresentados os comportamentos maternos e do recémnascido desejáveis e outros indicativos de problemas. Embora esse protocolo tenha sido amplamente divulgado e empregado no treinamento de equipes de maternidade em manejo do aleitamento materno, não foram encontrados estudos sobre a prevalência de dificuldades aferidas com este instrumento. Este trabalho teve como objetivo dimensionar o grupo de mães/recém-nascidos com necessidades especiais de apoio para um início bem sucedido do aleitamento materno, mediante aplicação de protocolo preconizado pelo UNICEF, e verificar práticas assistenciais associadas com dificuldades no aleitamento materno.

\section{Metodologia}

O estudo foi realizado na maternidade do Hospital Regional da Associação Botucatuense de Hospitais Sorocabana, responsável pelo atendimento a cerca de 50\% dos partos considerados de baixo risco do município de Botucatu, cidade de médio porte (cerca de 110.000 habitantes), situada na região Centro-Sul do estado de São Paulo. Esta maternidade é a única do município que atende ao parto de baixo risco pelo Sistema Único de Saúde.

Foi estudada uma amostra formada por 50 duplas mãe/ recém-nascido, selecionadas mediante sorteio dentre as parturientes atendidas no período de junho a novembro de 1998, durante visitas realizadas em dias da semana e períodos (manhã, tarde, noite) variados, de acordo com a disponibilidade de uma das pesquisadoras. A comparação de características da amostra com a população assistida na maternidade em 2000, ano mais próximo da coleta de dados deste estudo, para o qual existem dados no SINASC Sistema de Informações sobre Nascidos Vivos -, indicou que esta pode ser considerada representativa. A média de idade das mães $(24,0$ anos na amostra versus 24,4 na população), a proporção de adolescentes $(24,0 \%$ versus $22,1 \%$ ), de partos cesarianos ( $30 \%$ versus $28,8 \%$ ) e de mães com companheiro ( $86 \%$ versus $80,8 \%$ ) foram muito semelhantes. O peso médio das crianças também não diferiu muito (3.132g versus $3.226 \mathrm{~g}$ ).

As entrevistas foram realizadas por uma das autoras (CRHC) durante estágio do curso de graduação em enfermagem na maternidade. Os dados foram obtidos por meio da observação direta do fenômeno de interesse: comportamentos do binômio durante a mamada. Para guiar a observação e avaliar os comportamentos maternos e dos neonatos, empregou-se protocolo (Figura 1) difundido pelo UNI$\mathrm{CEF}^{11}$, o qual contém uma série de comportamentos classificados em favoráveis à amamentação, ou sugestivos de dificuldades, referentes à posição corporal da mãe e do recém-nascido durante a mamada, às respostas da dupla ao iniciarem a mamada, à eficiência da sucção, ao envolvimento afetivo entre a mãe e seu filho, às características anatômicas da mama e à duração e forma como se dá o encerramento da mamada. Considerou-se mamada o episódio completo, isto é, o período desde a decisão da mãe de colocar o recém-nascido para mamar até seu encerramento. Todos os binômios foram observados quando se encontravam no alojamento conjunto, e os recém-nascidos tinham entre 18 e 30 horas de vida

Além da observação da mamada, foram coletados dados sobre antecedentes gestacionais, tipo de parto e outros aspectos da assistência e dos cuidados dirigidos ao neonato e à puérpera, pesquisados nos prontuários da criança e da mãe, e também obtidos através de informações fornecidas pelos profissionais responsáveis pela assistência às duplas.

Esta pesquisa foi aprovada pelo Comitê de Ética em Pesquisa da Faculdade de Medicina de Botucatu.

Após revisão, os dados foram armazenados em banco construído no programa Excel/97 ${ }^{12}$. Para análise, empregou-se o programa SPSS for Windows, versão 6.0 $0^{13}$. Realizou-se inicialmente a caracterização das mães, de seus filhos e das práticas assistenciais a eles dirigidas na maternidade. A seguir, computou-se a freqüência de comportamentos desfavoráveis para cada aspecto da mamada investigada e, de acordo com o número de comportamentos negativos observados, foram criados escores de avaliação. $\mathrm{Na}$ Tabela 1, apresentam-se os critérios empregados na criação e classificação destes escores (bom, regular, ruim). Finalmente, foram realizadas algumas análises complementares, mediante comparação das diferenças entre proporções e análise de regressão linear, dirigidas a identificar fatores associados aos escores desfavoráveis. As associações foram submetidas a teste de significância estatística ( $\chi^{2}$ Pearson e teste $F$ ), adotando-se $p<0,05$ como nível crítico. 


\section{Resultados}

A idade materna variou de 15 a 43 anos, média de $24 \pm$ 6,2 anos; $24 \%$ (12 mães) das mães eram adolescentes; $86 \%$ (43 mães) viviam com companheiro; $92 \%$ (46 mães) havi- am passado por pelo menos seis consultas pré-natais, nenhuma por menos de duas consultas; 70\% (35 mães) dos partos foram vaginais.

\section{Comportamentos favoráveis}

\section{Comportamentos indicativos de dificuldades}

\section{Posição}

Mãe relaxada e confortável

Corpo e cabeça do bebê tocando o peito

Queixo do bebê tocando o peito

Nádegas do bebê apoiadas

Escore posição 1

Mãe com ombros tensos e inclinada sobre o bebê

Corpo do bebê distante do da mãe

O bebê está com o pescoço virado

O queixo do bebê não toca o peito

Só ombros/cabeça apoiados

Escore posição 2

\section{Respostas}

O bebê procura o peito quando sente fome

O bebê roda e busca o peito

O bebê explora o peito com a língua

Bebê calmo e alerta ao peito

Bebê mantém a pega da aréola

Sinais de ejeção de leite (vazamento, cólicas uterinas, fisgadas)

Escore resposta 1

Nenhuma resposta ao peito

Nenhuma busca observada

O bebê não está interessado no peito

Bebê irrequieto ou chorando

Bebê não mantém a pega da aréola

Nenhum sinal de ejeção de leite

Escore resposta 2

\section{Estabelecimento de laços afetivos}

Mãe segura o bebê no colo com firmeza

Mãe e bebê mantém contato visual

Grande quantidade de toques mãe/filho

Escore afetivo 1

Mãe segura o bebê nervosamente, sacudindo-o, tremendo ou fracamente

$\square$ Nenhum contato ocular mãe/filho

Mãe e bebê quase não se tocam

Escore afetivo 2

\section{Anatomia}

Mamas macias e cheias antes da mamada

Mamilos projetando-se para fora

Tecido mamário com aparência saudável

Mamas com aparência arredondada

Escore anatomia 1

Mamas ingurgitadas e duras

Mamilos planos ou invertidos

Tecido mamário com escoriações, fissuras, vermelhidão

Mamas esticadas ou caídas

Escore anatomia 2

\section{Sucção}

Boca bem aberta

Lábio inferior projeta-se para fora

Língua do bebê assume a forma de um cálice

ao redor do bico do peito

Bochechas de aparência arredondada

Sucção lenta e profunda com períodos de atividade e pausa

É possível ver e/ou ouvir a deglutição

Escore sucção 1

Boca quase fechada, fazendo um bico para a frente Lábio inferior virado para dentro

Não se vê a língua do bebê

Bochechas tensas ou encovadas

Sucções rápidas com estalidos

Pode-se ouvir barulho altos, mas não a deglutição

Escore sucção 2

Figura 1 - Formulário de observação e avaliação de mamada 
Tabela 1 - Critérios para classificação dos escores empregados na avaliação da mamada segundo cada aspecto avaliado

\begin{tabular}{lcccc}
\hline Aspectos avaliados & $\begin{array}{c}\mathbf{N}^{\mathbf{0}} \text { de } \\
\text { comportamentos } \\
\text { negativos investigados }\end{array}$ & \multicolumn{2}{c}{$\begin{array}{c}\text { Comportamentos } \\
\text { negativos observados / } \\
\text { Classificação dos escores }\end{array}$} \\
\cline { 3 - 5 } & 05 & $0-1$ & $2-3$ & $4-5$ \\
\hline Pom & $0-1$ & $2-3$ & $4-6$ \\
Regular & Ruim \\
Adequa mãe/criança & 06 & $0-1$ & $2-3$ & $4-6$ \\
Anatomia da dupla & 06 & 0 & 1 & $2-4$ \\
Afetividade da sucção & 04 & 0 & 1 & $2-3$ \\
\hline
\end{tabular}

Os neonatos apresentaram peso médio ao nascimento de $3.132 \mathrm{~g} \pm 428 \mathrm{~g}$, idade gestacional média de $39,3 \pm 1,5$. A proporção de bebês com baixo peso ao nascimento $(<2.500 \mathrm{~g})$ foi de $8 \%$ (4 recém-nascidos). A baixa vulnerabilidade dos recém-nascidos estudados também pôde ser aferida pela pequena proporção que permaneceu algum tempo em incubadora ( $8 \%$; 4 bebês) e em fototerapia (10\%; 5 bebês).

Apenas duas crianças mamaram na sala de parto; o início precoce das mamadas - até duas horas após o parto ocorreu para 36\% delas (18 crianças), e o início tardio (após sete horas de vida), para 12 recém-nascidos (24\%). Cerca de 36\% das mães (18 mães) consideraram satisfatória esta primeira mamada. De acordo com as anotações nas folhas de evolução, 29 recém-nascidos $(58 \%)$ receberam soro glicosado, e 9 (18\%), leite de outra espécie, sempre por meio de mamadeira.

Os resultados da avaliação dos comportamentos da mãe e do recém-nascido na mamada observada constam da Figura 2. Nota-se que grande parte dos binômios apresentou escores adequados (bom) indicativos de início satisfatório da amamentação nos diversos aspectos analisados. Computando-se os escores regulares e ruins, a freqüência de binômios com comportamentos sugestivos de dificuldades com o início da amamentação variou entre $18 \%$ (9 duplas) e 34\% (17 duplas), conforme o aspecto em análise. A ocorrência de escores mais desfavoráveis (escore ruim), ou seja, muitos comportamentos sugestivos de dificuldades que podem conduzir ao desmame precoce, situou-se entre $2 \%$ (1 dupla) e $22 \%$ (11 duplas). Os piores resultados foram relativos à posição corporal da mãe e do recém-nascido durante a mamada, pois $22 \%$ das duplas apresentaram quatro ou cinco dos cinco comportamentos negativos investigados.

A observação de aspectos da anatomia das mamas revelou que $30 \%$ das mães (somando-se 14 mães com escore regular e 1 mãe com escore ruim) apresentavam algum tipo de lesão no mamilo (escoriações, fissuras mami- lares) e/ou ingurgitamento mamário no momento da observação.

Escores ruins também ocorreram para 12\% (6 duplas) dos binômios quando se avaliaram determinadas respostas negativas, tais como: neonato inquieto e agitado, não conseguindo manter a pega da aréola, e mãe sem sinais de ejeção de leite (vazamento, "fisgada na mama" e cólicas uterinas).

Cerca de $6 \%$ (3 crianças) dos recém-nascidos apresentaram escores ruins na avaliação da sucção, isto é, quatro a seis comportamentos negativos, tais como: lábio inferior virado para dentro, sem visualização da língua, bochechas tensas ou encovadas e criança sugando rapidamente, podendo-se ouvir apenas estalos, e não a deglutição.

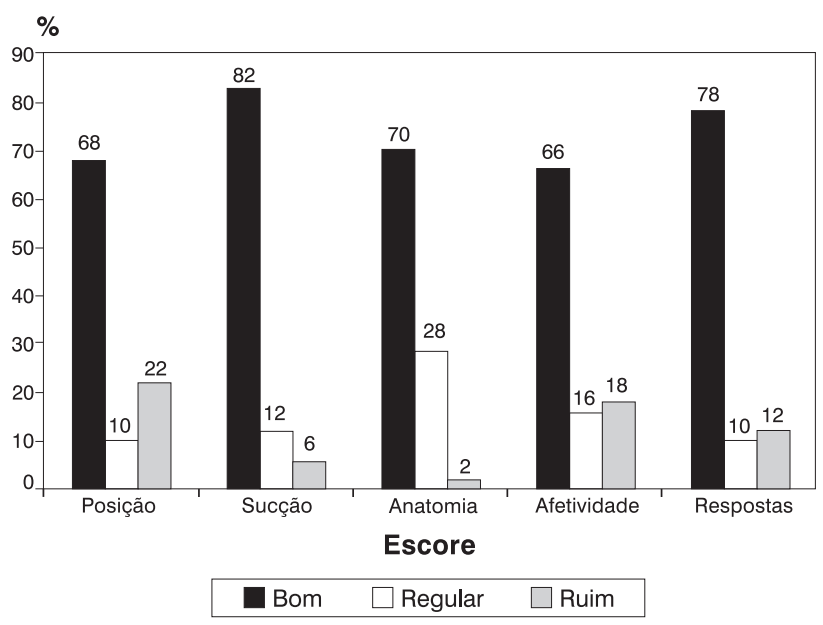

Figura 2 - Distribuição (\%) das duplas mãe/recém-nascido de acordo com os escores relativos à posição corporal, sucção, anatomia das mamas, afetividade e respostas da dupla - Botucatu, 1998 
Com relação à avaliação dos comportamentos indicativos do estabelecimento de laços afetivos entre a mãe e seu filho, também foi detectada significativa freqüência (18\%; 9 duplas) de escores insatisfatórios, ou seja, mães que quase não tocavam nem estabeleciam comunicação visual com a criança, mantendo-se distantes do recém-nascido.

$\mathrm{O}$ tipo de parto associou-se significativamente com os escores relativos à avaliação da posição corporal $(p<0,01)$ e aos comportamentos afetivos ( $\mathrm{p}<0,01)$. As duplas mães/ bebês com parto operatório apresentaram escores significativamente mais desfavoráveis referentes à posição corporal da mãe e do recém-nascido e ao envolvimento afetivo. Com relação aos escores para adequação da sucção e das respostas do recém-nascido, e para aspectos da anatomia das mamas, não houve associação significativa com o tipo de parto. Tais dados encontram-se na Tabela 2.

Na Tabela 3, são apresentados os resultados da investigação da existência de associação entre o uso de líquidos suplementares e escores desfavoráveis. O consumo de soro

Tabela 2 - Distribuição das duplas mãe/recém-nascido em relação aos escores em cada aspecto da mamada segundo tipo de parto - Botucatu, 1998

\begin{tabular}{|c|c|c|c|c|}
\hline \multirow[t]{3}{*}{ Escores } & \multicolumn{4}{|c|}{ Tipo de parto } \\
\hline & \multicolumn{2}{|c|}{ Vaginal } & \multicolumn{2}{|c|}{ Cesariana } \\
\hline & $\mathbf{N}$ & $\%$ & $\mathbf{N}$ & $\%$ \\
\hline \multicolumn{5}{|l|}{ Posição * } \\
\hline Bom & 26 & 74,3 & 08 & 53,3 \\
\hline Regular & 04 & 11,4 & 01 & 6,7 \\
\hline Ruim & 05 & 14,3 & 06 & 40,0 \\
\hline Total & 35 & 100,0 & 15 & 100,0 \\
\hline \multicolumn{5}{|c|}{ Afetividade ${ }^{\dagger}$} \\
\hline Bom & 27 & 77,1 & 06 & 40,0 \\
\hline Regular & 02 & 5,7 & 06 & 40,0 \\
\hline Ruim & 06 & 17,2 & 03 & 20,0 \\
\hline Total & 35 & 100,0 & 15 & 100,0 \\
\hline \multicolumn{5}{|c|}{ Adequação da sucção $\ddagger$} \\
\hline Bom & 30 & 85,8 & 11 & 73,3 \\
\hline Regular & 4 & 11,4 & 2 & 13,3 \\
\hline Ruim & 1 & 2,8 & 2 & 13,4 \\
\hline Total & 35 & 100,0 & 15 & 100,0 \\
\hline \multicolumn{5}{|c|}{ Respostas do recém-nascido ${ }^{\S}$} \\
\hline Bom & 26 & 74,2 & 13 & 86,7 \\
\hline Regular & 4 & 11,5 & 1 & 6,7 \\
\hline Ruim & 5 & 14,3 & 1 & 6,6 \\
\hline Total & 35 & 100,0 & 15 & 100,0 \\
\hline \multicolumn{5}{|c|}{ Anatomia das mamas $\|$} \\
\hline Bom & 24 & 68,6 & 11 & 73,3 \\
\hline Regular & 10 & 28,6 & 4 & 26,7 \\
\hline Ruim & 1 & 2,8 & 0 & 0 \\
\hline Total & 35 & 100,0 & 15 & 100,0 \\
\hline
\end{tabular}

Teste qui-quadrado (Pearson)

${ }^{*} p<0,01 ;{ }^{\dagger} p<0,01 ;{ }^{\ddagger} p=0,51$ (NS); $§ p=0,32$ (NS); $\| p=0,79$ (NS) glicosado associou-se com piores escores relativos às respostas do recém-nascido ao ser levado para mamar ( $\mathrm{p}=$ $0,03)$, e permaneceu no limiar da significância estatística em relação ao escore de posição corporal das duplas ( $\mathrm{p}=$ 0,056 ); bebês que receberam fórmula láctea apresentaram piores escores referentes à posição corporal da dupla $(\mathrm{p}=0,03)$ e à adequação da sucção $(\mathrm{p}=0,02)$.

De acordo com as análises de regressão linear univariadas realizadas, não houve associação entre características dos bebês - idade gestacional, peso ao nascimento e idade na primeira mamada - e os diferentes escores, dados apresentados na Tabela 4.

\section{Discussão}

A presente pesquisa apontou que, somando-se as freqüências de escores regulares e ruins, de 18 a $34 \%$ dos binômios mãe/recém-nascido apresentaram alguma dificuldade com o início da amamentação em pelo menos um dos aspectos da mamada observada. Esta é a magnitude do grupo que, na maternidade estudada, necessita de ações intensivas de apoio ao início do aleitamento materno.

Não foram encontrados outros estudos que tenham pesquisado a prevalência de comportamentos indicativos de problemas com o início da amamentação utilizando o protocolo UNICEF. Há na literatura pesquisada apenas um estudo, realizado no alojamento conjunto do Hospital Guilherme Álvaro, um Centro de Referência em Lactação e Hospital Amigo da Criança, que investigou dificuldades precoces com a amamentação, porém utilizando outros instrumentos para a observação das duplas e avaliação da mamada e da eficiência da sucção ${ }^{14}$. A frequiência de duplas com dificuldades, cerca de $13 \%$, foi menor do que a observada no presente estudo. Entretanto, deve-se apontar que, no referido hospital, as práticas assistenciais são mais adequadas ao início bem sucedido do aleitamento materno do que na maternidade onde a presente pesquisa foi realizada.

Os problemas precoces detectados entre os binômios referem-se principalmente à amamentação em posição que dificulta a pega adequada e pode causar traumas mamilares, mamadas pouco eficientes e o não esvaziamento completo da mama, com conseqüências negativas para a produção de leite e para o crescimento do bebê ${ }^{11}$. De fato, muitas mães amamentavam em posição desfavorável, ou seja, mantinham o corpo da criança distante do seu, ficando inclinadas sobre ela; seguravam-na apoiando apenas o ombro e a cabeça. Grande parte dos recém-nascidos precisava virar o pescoço para mamar, conseqüentemente, o queixo não tocava o seio materno. Todas estas condições tendem a dificultar a pega adequada ${ }^{11}$.

A associação detectada entre piores escores relativos à posição da dupla e parto cirúrgico pode expressar uma relação causal. A cesariana altera as respostas endócrinas na mãe e no bebê no período imediato após o parto, além de 
Tabela 3 - Distribuição das duplas mãe/recém-nascido segundo escores de avaliação de mamada e recebimento de líquidos suplementares - Botucatu, 1998

\begin{tabular}{|c|c|c|c|c|c|c|c|c|}
\hline \multirow{3}{*}{$\begin{array}{l}\text { Escore / } \\
\text { Aspecto avaliado }\end{array}$} & \multicolumn{4}{|c|}{ Fórmula láctea } & \multicolumn{4}{|c|}{ Soro glicosado } \\
\hline & \multicolumn{2}{|c|}{ Sim } & \multicolumn{2}{|c|}{ Não } & \multicolumn{2}{|c|}{ Sim } & \multicolumn{2}{|c|}{ Não } \\
\hline & $\mathbf{N}$ & $\%$ & $\mathbf{N}$ & $\%$ & $\mathbf{N}$ & $\%$ & $\mathbf{N}$ & $\%$ \\
\hline \multicolumn{9}{|l|}{ Posição } \\
\hline Bom & 5 & $(55,6)$ & 29 & $(70,8)^{a}$ & 17 & $(58,6)$ & 17 & $(80,9)^{\mathrm{c}}$ \\
\hline Regular & - & $(0,0)$ & 5 & $(12,1)$ & 4 & $(13,8)$ & 1 & $(4,8)$ \\
\hline Ruim & 4 & $(44,4)$ & 7 & $(17,1)$ & 8 & $(27,6)$ & 3 & $(14,3)$ \\
\hline \multicolumn{9}{|l|}{ Respostas } \\
\hline Bom & 5 & $(55,6)$ & 34 & $(82,9)$ & 20 & $(69,0)$ & 19 & $(90,5)^{d}$ \\
\hline Regular & 1 & $(11,1)$ & 4 & $(9,8)$ & 5 & $(17,2)$ & - & $(0,0)$ \\
\hline Ruim & 3 & $(33,3)$ & 3 & $(7,3)$ & 4 & $(13,8)$ & 2 & $(9,5)$ \\
\hline \multicolumn{9}{|l|}{ Sucção } \\
\hline Bom & 6 & $(66,7)$ & 35 & $(85,3)^{b}$ & 24 & $(82,8)$ & 17 & $(81,0)$ \\
\hline Regular & 3 & $(33,3)$ & 4 & $(9,8)$ & 5 & $(17,2)$ & 2 & $(9,5)$ \\
\hline Ruim & - & $(0,0)$ & 2 & $(4,9)$ & - & $(0,0)$ & 2 & $(9,5)$ \\
\hline \multicolumn{9}{|l|}{ Anatomia } \\
\hline Bom & 6 & $(66,7)$ & 29 & $(70,8)$ & 18 & $(62,1)$ & 17 & $(80,9)$ \\
\hline Regular & 2 & $(22,2)$ & 12 & $(29,2)$ & 10 & $(34,5)$ & 4 & $(19,1)$ \\
\hline Ruim & 1 & $(11,1)$ & - & $(0,0)$ & 1 & $(3,4)$ & - & $(0,0)$ \\
\hline \multicolumn{9}{|l|}{ Afetividade } \\
\hline Bom & 4 & $(44,5)$ & 29 & $(70,8)$ & 17 & $(58,6)$ & 16 & $(76,2)$ \\
\hline Regular & 2 & $(22,2)$ & 6 & $(14,6)$ & 7 & $(24,2)$ & 1 & $(4,8)$ \\
\hline Ruim & 3 & $(33,3)$ & 6 & $(14,6)$ & 5 & $(17,2)$ & 1 & $(19,0)$ \\
\hline
\end{tabular}

provocar dor na mãe e maior sonolência no binômio, condições que retardam e impõem dificuldades nas primeiras mamadas 15,18 .

Não foram encontrados na literatura estudos que tenham investigado especificamente a relação entre parto cirúrgico e comportamentos maternos e do recém-nascido durante a mamada. Porém, vários estudos investigaram a relação entre tipo de parto e incidência e duração do aleitamento materno. No Sul do Brasil, um estudo apontou risco aumentado de desmame completo no primeiro mês de vida em mães que tiveram seus filhos por cesariana eleti$\mathrm{va}^{15}$. Outro estudo, também no Sul do Brasil, encontrou

Tabela 4 - Associação* entre características do recém-nascido e escores de avaliação de mamada Botucatu, 1998

\begin{tabular}{|c|c|c|c|c|c|c|c|c|c|}
\hline \multirow[t]{2}{*}{ Escores } & \multicolumn{3}{|c|}{$\begin{array}{c}\text { Idade } \\
\text { gestacional }\end{array}$} & \multicolumn{3}{|c|}{$\begin{array}{c}\text { Variáveis } \\
\text { Peso } \\
\text { ao nascimento }\end{array}$} & \multicolumn{3}{|c|}{$\begin{array}{c}\text { Idade na } \\
\text { primeira mamada }\end{array}$} \\
\hline & $\mathbf{R}^{2}$ & $\mathbf{F}$ & $\mathbf{p}$ & $\mathbf{R}^{2}$ & $\mathbf{F}$ & $\mathbf{p}$ & $\mathbf{R}^{2}$ & $\mathbf{F}$ & $\mathbf{p}$ \\
\hline Posição & 0,0032 & 0,15 & 0,70 & 0,007 & 0,37 & 0,54 & 0,012 & 0,59 & 0,45 \\
\hline Respostas & 0,019 & 0,94 & 0,34 & 0,011 & 0,53 & 0,47 & 0,0055 & 0,26 & 0,61 \\
\hline Sucção & 0,00048 & 0,023 & 0,88 & 0,0056 & 0,27 & 0,61 & 0,009 & 0,42 & 0,52 \\
\hline Anatomia da mama & 0,012 & 0,58 & 0,45 & 0,0007 & 0,003 & 0,95 & 0,00013 & 0,0063 & 0,94 \\
\hline Afetividade & 0,012 & 0,57 & 0,45 & 0,021 & 1,03 & 0,31 & 0,01941 & 0,95 & 0,33 \\
\hline
\end{tabular}

* Mediante análise de regressão linear univariada, todos resultados não são significantes. 
menor prevalência de aleitamento materno aos seis meses em crianças nascidas de parto cirúrgico ${ }^{16}$. Esta associação também foi encontrada em pesquisa realizada no México ${ }^{17}$. Em Campinas (SP), estudo realizado no alojamento conjunto de uma maternidade-escola verificou que a cesariana constituiu um dos principais fatores de risco para a oferta de líquidos suplementares aos neonatos ${ }^{18}$. É possível que uma das maneiras pelas quais a cesariana afete negativamente a duração do aleitamento materno seja justamente aumentando a ocorrência de dificuldades em seu início, tal como detectado no presente estudo.

Além das dificuldades relativas à posição das duplas durante a mamada, proporção significativa de mães (18\%) apresentou um padrão de interação mãe/neonato pobre em comportamentos indicativos de acolhimento e autoconfiança. Estas dificuldades também foram mais freqüentes quando o parto foi cirúrgico. Esta associação também pode ser explicada pelas diferenças nas respostas maternas e do recém-nascido após a cesariana. Particularmente, o uso de anestésicos e a dor afetam a interação mãe/bebê $\hat{e}^{19}$.

Estes resultados, apoiados na literatura acima citada, permitem sugerir que as dificuldades com o início da amamentação sejam incluídas como mais um possível desfecho negativo da prática, ainda presente em hospitais brasileiros, de realizar cesarianas desnecessárias.

Outro resultado muito significativo refere-se às práticas de cuidado do recém-nascido. Apesar de ativamente combatidas há mais de uma década, detectou-se alta freqüência de práticas desaconselhadas na rotina da maternidade, tais como: demora na primeira mamada e a conseqüente oferta de líquidos, tanto soro glicosado quanto complementos lácteos, e o uso de mamadeiras para esta oferta. Sabe-se que tais práticas são desnecessárias, na maioria das vezes, para crianças sadias como as incluídas neste estudo, e constituem procedimentos desfavoráveis ao aleitamento materno, principalmente por levarem à confusão de bico ${ }^{11}$. De fato, o uso de fórmula láctea associou-se com maior freqüência de escores ruins na avaliação da sucção, e o uso de soro glicosado com piores escores na avaliação das respostas do recém-nascido.

Os recém-nascidos que recebem fórmula láctea estão ainda expostos a outros efeitos adversos. O contato precoce do neonato com proteína de leite de outra espécie constitui significativo fator de risco para doenças alérgicas e autoimunes, tais como bronquite, dermatite atópica, intolerância ou alergia ao leite de vaca e diabetes tipo I. O uso de outros líquidos, mesmo soro glicosado, rompendo o aleitamento exclusivo, eleva o risco de morbidade, especialmente diarréia, pneumonia e otites ${ }^{20-22}$.

Finalizando, cabe apontar que o protocolo de observação utilizado neste estudo, com a adoção de escores para avaliação da freqüência de comportamentos desfavoráveis, revelou-se procedimento viável, simples, não requerendo profissional especialmente capacitado em manejo do aleitamento materno, e poderia ser adotado rotineiramente para todos os recém-nascidos. Quando não houver recursos humanos disponíveis para tal, a utilização do protocolo poderia concentrar-se em neonatos nascidos de parto cirúrgico e naqueles que, por razões aceitáveis ou não, receberam líquidos suplementares. Identificados os binômios com maiores dificuldades, um membro da equipe, treinado em manejo clínico e aconselhamento da amamentação, passaria a atuar.

Além de permitir a identificação de mães e bebês com maiores dificuldades para iniciar a amamentação, o protocolo de observação de mamada e o cômputo de escores nele baseados permitiriam incluir, de modo objetivo, o desempenho na amamentação nos critérios de alta da maternidade. Escores muito ruins em diversos aspectos da mamada poderiam determinar o prolongamento da internação até que as dificuldades fossem minimizadas, ou indicar a realização de intervenções de apoio em domicílio. Entretanto, há necessidade de estudo de validação do protocolo em nosso meio.

\section{Referências bibliográficas}

1. WHO - World Health Organization. World Health Assembly 47.5. Geneva: WHO; 1994.

2. Carvalhaes MABL, Parada MCGL, Manoel CM, Venâncio SY. Diagnóstico da situação do aleitamento materno em área urbana do Sudeste do Brasil: utilização de metodologia simplificada. Rev Saúde Pública 1998;32:430-6.

3. Aldair LS, Popkin BM, Guilkey DK. The duration of breastfeeding: how is it affected by biological, sociodemographic, heath sector, and food industry factors? Demography 1993;30: 63-80.

4. Winikoof B, Baer EC. The obstetrician's opportunity: translating "breast is best" from theory to practice. Am J Obstet Gynecol 1980;138:105-17.

5. Giugliani ERJ, Lovel H, Ebraim GL. Attitudes, practices and knowledge of health professionals on breast-feeding in Kingston, Jamaica. J Trop Pediatr 1988;34:169-73.

6. Saadeh R, Akré J. Ten steps to successful breastfeeding: a summary of the rationale and scientific evidence. Birth 1996;23: 154-60.

7. Schy DS, Malaga CF, Mendelson SG, Race KE, LudwigBeymer P. The effects of in-hospital lactation education on breastfeeding practice. J Hum Lact 1996;12:117-22.

8. Patton CB, Beaman M, Sarn C, Lewinski C. Nurses' attitudes and behaviors that promote breast-feeding. J Hum Lact 1996; 12:111-5.

9. Grant JP. Solicita-se apoio aos médicos. Boletim da Iniciativa Hospital Amigo da Criança; 1994.

10. OMS/UNICEF. Proteção, promoção e apoio ao aleitamento materno: o papel essencial dos serviços materno-infantis. Genebra: Declaração conjunta OMS/UNICEF; 1989.

11. UNICEF. Breastfeeding management and promotion in a babyfriendly hospital: an 18-hour course for maternity staff. New York: UNICEF; 1993.

12. Excel-97 [computer program]. Version 1997. USA: Microsoft; 1997.

13. SPSS- Statistical Package [computer program]. Version 6.0. USA: SPSS inc.; 1993. 
14. Sanches, MTC. Dificuldades iniciais na amamentação: enfoque fonoaudiológico [dissertação]. São Paulo: Faculdade de Saúde Pública; 2000.173p.

15. Ford K, Labbok M. Who is breast-feeding? Implications of associated social and biomedical variables for research on consequences of methods of infant feeding. Am J Clin Nutr 1990;52:451-6.

16. Victora CG, Huttly SRA, Barros FC, Vaughan JP. Cesarean section and duration of breast-feeding among brazilians. Arch Dis Child 1990;65:632-4.

17. Weiderpass E, Barros, FC, Victora CG, Tomasi E, Halpern R. Incidência e duração da amamentação conforme o tipo de parto: estudo longitudinal no Sul do Brasil. Rev Saúde Pública 1998; 32:225-31.

18. Pérez-Escamilla R, Maulén-Radovan I, Dewey KG. The association between cesarean delivery and breast-feeding outcomes among Mexican women. Am J Public Health 1996; $86: 832-6$.

19. Pinto LM, Vítolo MR, Gírio LT, Leon MRAC, Zaguri MCF, Fernandes NM, et al. Aleitamento materno exclusivo em alojamento conjunto: avaliação da incidência e das causas do uso de fórmulas. Rev Ciências Méd PUCCAMP 1996;5:63-8.

20. Brazelton TB. Effect of maternal medication on the neonate and his behaviour. J Pediatr 1961;58:513-54.
21. Duncan B, Ey J, Holberg CJ, Wright AL, Martinez FD, Taussig LM. Exclusive breastfeeding for at lest 4 months protect against otitis media. Pediatrics 1993;9:867-72.

22. Victora C, Smith PG, Vaughan JP, Nobre LC, Lombardi C, Teixeira AM, et al. Evidence for protection by breastfeeding against infant death from infection disease in Brazil. Lancet 1987;2:319-22.

23. Woolridge MW, Phil D, Baum JD. Recent advances in breastfeeding. Acta paediatr JPN 1993;35:1-12.

Endereço para correspondência

Dra. Maria Antonieta de Barros Leite Carvalhaes

Departamento de Enfermagem

Faculdade de Medicina de Botucatu

Universidade Estadual Paulista

E-mail: carvalha@fmb.unesp.br 